\title{
An Overview of the Drug Susceptibility Testing for Tuberculosis
}

\section{Vikas Jha ${ }^{1 *}$, BS Ajit Kumar ${ }^{2}$, Sampurna Panigrahi ${ }^{3}$, Gayatri Nair ${ }^{3}$ and Nikitha Bangera ${ }^{3}$}

${ }^{1}$ National Facility of Biopharmaceutical, Mumbai, India

${ }^{2}$ V.P.M's R. Z. Shah College Mulund, India

${ }^{3}$ School of Biotechnology and Bioinformatics, DY Patil deemed to be University, CBD

Belapur, Navi Mumbai, India

*Corresponding Author: Vikas Jha, National Facility for Biopharmaceuticals,

Mumbai, India.

DOI: $10.31080 /$ ASMI.2020.03.0653
Received: June 30, 2020

Published: July 25, 2020

(C) All rights are reserved by Vikas Jha., et al.

\section{Abstract}

There in an increase in demand for reliable, inexpensive and rapid drug susceptibility assay because of expanding anti-tuberculosis drug-resistant Mycobacterium tuberculosis necessitating the need for appropriate treatment. One of the major challenges being faced is the lack of resources and the limiting of reliable drug susceptibility test meeting acceptable levels only for isoniazid and rifampicin. In this article, an overview of different drug susceptibility testing and assays is detailed and the advantages and disadvantages highlighted. It discusses the perspective on conventional methods which have paved the way for modern DSTs along with the advancements made in the conventional methods.

Keywords: Drug Susceptibility; Anti-tuberculosis; Mycobacterium tuberculosis; Conventional DST's

\section{Introduction}

Tuberculosis is one of the top 10 causes of mortality worldwide and was declared a global emergency nearly two and a half decades ago [1,2]. The causal agent being Mycobacterium tuberculosis, is a rod-shaped, acid-fast bacterium, usually transmitted by aerosol infection and is the leading cause of death from a single infectious agent $[2,3]$. An estimation of nearly 10.0 million (range, 9.0 - 11.1 million) people have fallen sick globally due to tuberculosis in 2018 though the number has remained fairly stable in recent years. There is a decline of $1.6 \%$ per year in the period 2000 - 2018 and 2.0\% between 2017 and 2018 in the tuberculosis incidence rate, the cumulative reduction being 6.3\% between 2015 and 2018.

Many cases of active tuberculosis are still not detected and are not treated in a timely manner, especially in developing countries. India and Indonesia are the two countries which rank first and third worldwide in terms of estimated incident cases per year

\section{POPULATION 20187604 MILLION}

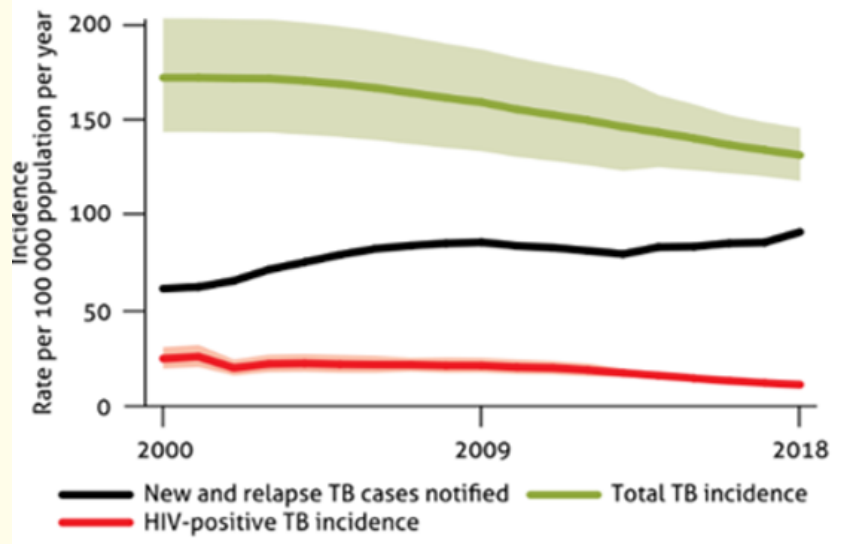

Figure 1: Global tuberculosis report 2019, WHO (Global report of cases). 
are primarily responsible for the increase in the global notifications of tuberculosis cases since 2013, observing an increase from 1.2 million cases to 2.0 million between 2013 and 2018 solely in India [2].

\section{POPULATION 20181353 MILLION}

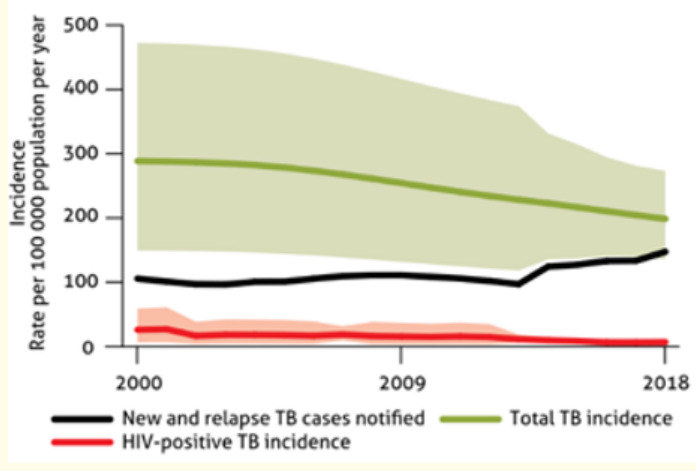

Figure 2: Global tuberculosis report 2019, WHO (India).

Prompt identification of new incidents and rapid implementation of effective treatment regiments to interpret transmission are two critical components of tuberculosis transmission. The standard treatment combines isoniazid (INH), rifampin (RIF), pyrazinamide (PZA) and ethambutol (EMB) which are the four first-line antibiotics, which when properly administered renders patients suffering from tuberculosis noncontagious. Inefficient treatment can lead to drug resistance bacilli (acquired resistance) and those resistant organisms can be transmitted to other individuals (primary resistance).

Multidrug-resistant (MDR) TB, i.e. bacilli resistant to the firstline drugs isoniazid and rifampin (rifampicin) has garnered much attention over the years along with the emergence of the more deadly extensively drug-resistant tuberculosis (XDR) [4].

Drug-resistant tuberculosis continues to be a public health threat. In 2018, there were about half a million new cases of rifampicin-resistant tuberculosis (of which $78 \%$ had multidrugresistant tuberculosis). Extensively drug-resistant TB (XDR-TB) is defined as MDR-TB plus resistance to at least one of the fluoroquinolones and one of the injectable agents (second-line drugs) used in MDR-TB treatment regimens [2].
The expanding problem of resistance in Mycobacterium tuberculosis has driven a requirement for quick, cheap, and easy techniques to detect resistance. Various methods have been developed over the years to make susceptibility testing feasible. The determination of drug susceptibility of Mycobacterium tuberculosis can be ascertained by the examination of a medium comprising an anti-tuberculosis drug for growth or metabolic inhibition, or via detection of the mutation in genes associated to drug action at the molecular level. On the basis of technical standpoint, the detection of drug susceptibility by growth or metabolic inhibition can be followed out by means of observing drug-free and drug-containing media consisting macroscopic growth or by metabolic activity or products detection and measurement. It can also be carried out by lysis with mycobacteriophage or genetic mutation detection availing molecular techniques [5]. Molecular tests promise a more rapid drug resistance detection with a disadvantage of expenses.

Mycobacterium tuberculosis isolates can take weeks to months to completely define the drug resistance pattern via conventional phenotypic methods due to the very slow growth of this bacterium. Drug susceptibility testing (DST) using the Lowenstein Jensen (LJ) include proportion method, resistant ratio method and the absolute concentration method which for major anti-tuberculosis drugs is fairly well standardised with clinical samples. It is a timeconsuming phenotypic DST method, taking up to 6 - 8 weeks to recover Mycobacteria or discriminate negative samples [6].

Egg- or agar-based media for conventional culture methods are still frequently employed by many countries. Proportion method amid conventional methods is the most favoured option, but in matters of frequency, absolute concentration method is also often used due to its technical simplicity of inoculum preparation and result interpretation.

Numerous techniques have been devised for early detection of growth inhibition to shorten the turnaround time (TAT) and make case management more convenient. The most often used systems are detection oare carbon dioxide production detection systems, such a BACTEC 460 or MB/Bact, and consumption of oxygen, such as Mycobacteria Growth Indicator Tube (MGIT). BACTEC 460 TB despite reducing TAT to 1 - 2 weeks it places higher demands on equipment to be routinely used in poor-resource countries also utilising substrates and expensive technology [8,9]. 
Colorimetric methods is another in the developmental stage of DST relying on oxidation-reduction indicators like resazurin or tetrazolium bromide [10]. Phage based technique is a method developed combining phenotypic assays and deploying bacteriophage to introduce into any viable isolate of $M$. tuberculosis the firefly luciferase gene (Fflux) [8]. Detection of a low-level multiplication of $M$. tuberculosis by particle-counting immunoassay can also curtail TAT. These techniques are quite difficult to be implemented in developing countries and countries where these are required indispensably due to the drawbacks of being expensive, technically complex and absence of appropriately trained human resources.

Detection of gene mutations related to resistance using molecular techniques including hybridization of amplified gene segments or other PCR-based methods can be utilized but as primary amplification is required for molecular techniques when followed on a routinely basis for long periods of time false results can be generated due to contaminating amplicons and/or chromosomal DNA [5].

Phenotypic method

Solid culture

In the 1960s, Cannetti., et al. described the first DST method for M. tuberculosis, classifying the test as direct and indirect where the direct test involves the sputum homogenate or other pathological material cultured directly on drug-containing medium, though the results obtained are not considered reliable. In indirect test the primary diagnostic culture was inoculated in a drug-containing medium. This was further classified into three main categories: (a) the absolute-concentration method; (b) the resistance-ratio method; and (c) the proportion method [11].

According to the method developed at the National Institute for Public Health and the Environment (RIVM), Bilthoven, The Netherlands in the absolute concentration method, a concentration series of anti-tuberculosis drugs are added to 7H10 medium distributed in 25-well plates. Furthermore, to check the sizes of the inocula and to compare the mycobacterial growth levels control is included in the presence of different concentrations of anti-tuberculosis drugs in a proportional manner. The determination of MIC can also be ascertained by this method [12].

In the resistance-ratio method, media containing two-fold dilution of the primary anti-tuberculosis drugs are prepared as parallel sets and one drop of bacillary suspension is spread on the surface of each drug-containing slopes of media of a series of concentration, with similar procedure followed with $\mathrm{H}_{37} \mathrm{Rv}$ strain. All tubes are incubated for 4 weeks at $37^{\circ} \mathrm{C}$ and weekly observed. The growth is defined as the presence of 20 or more colonies in the drug-containing media. The isolates are resistant when the growth appears on the media containing a given drug concentration in which control strain is susceptible. DST critical concentrations for second-line drugs have not yet been adequately validated for the resistance ratio and absolute concentration methods.

Among the three methods, the proportion method is the most commonly used method worldwide [13]. In the proportional method, Löwenstein-Jensen slants with critical drug concentrations are prepared for different anti-TB drugs. Parallel preparation of drugfree control media is carried out. The standardised culture suspension is diluted in sterile distilled water in different dilutions. From each dilution a drug-containing media is inoculated with one loopfull of bacillary suspension as well as controls of plain LJ media are inoculated with the respective diluted bacillary suspension. The slopes are observed after 28 days of incubation in $37^{\circ} \mathrm{C}$. Any colonies growing on drug-containing medium inoculated with the $10^{-1}$ dilution that equal or more the number of colonies growing on the control medium inoculated with the $10^{-3}$ dilution represents $1 \%$ or more of the test population. If the calculation was $1 \%$ or more then it is interpreted as resistant [14].

\section{Liquid culture}

In comparison to solid culture liquid culture reduces the turnaround time significantly for results and are around $10 \%$ more sensitive than solid media culture. The confirmation can be obtained within two weeks and can be used for susceptibility testing for both first-line and second-line drugs [13].

\section{BACTEC 460}

BACTEC 460 is a semi-automated, well-established broth-based method providing rapid detection of mycobacterium in a closed system. The introduction of the BACTEC 460 TB System revolutionized laboratory testing for mycobacteria and has established itself as the gold standard for culture and susceptibility testing [15].

The major drawback harboured by this system is the radiometric method used to detect the mycobacterial growth. BACTEC 460 generates radioactive waste due to usage of radioisotopes making 
disposal pose a considerable logistical problem as well an increase in expenses [16]. Another disadvantage of this method is its requirement for plating thus increasing the duration of incubation thereby elevating the risk of cross contamination [15]. Another disadvantage of this method is its requirement for plating thus increasing the duration of incubation thereby elevating the risk of cross contamination.

\section{BACTEC MGIT 960}

It is a fully automated, nonradiometric instrument which detects the growth of mycobacterium by exploiting the fluorescence of an oxygen sensor. In the study conducted by Enrico Tortoli., et al. 1999 the BACTEC MGIT 960 performance was compared to those of the radiometric BACTEC 460 instrument and egg-based Lowenstein-Jensen medium. The shortest time for detection was obtained with the BACTEC MGIT 960 system though the contamination rate observed was intermediate $(10.0 \%)$ to that of radiometric system (3.7\%) and the egg-based medium (17.0\%).

This system is comparable to BACTEC 460 eliminating two core problems posed by the system one being reducing needle puncture risk and overcoming the risk of disposal of radioactive waste. Further the advancements in automation of the MGIT 960 has led to easy and continuous monitoring of the positive fluorescence depending on the bacterial growth. It is a non-invasive and eliminates the requirement of labour thus dismissing the possibility of reading difficulties during the visual assessment of the tubes. The susceptibility can be automatically determined with the help of the threshold algorithms [17]. Although this system is faster than LJ culture the expenses are relatively high restricting their usage in low-income, heavy burden regions. Although this system is faster than LJ culture the expenses are relatively high restricting their usage in low-income, heavy burden regions.

\section{Colorimetric method}

It is a quantitative measurement of the susceptibility of $M$. tuberculosis against anti-tuberculosis agents. Diagnostic test for tuberculosis is either expensive (molecular methods and automated liquid-based culture systems) or slow (culture on solid media and biochemical tests). Therefore, an alternative method was developed which is rapid, quantitative, and nonradiometric and does not require the use of instrumentation [18].
Numerous low-cost colorimetric assays described are mainly based on the reduction of a coloured indicator added to the culture medium after the exposure of M. tuberculosis in in-vitro to different antibiotics. The detection of resistance is indicated by the change in colour of the indicator, which is directly proportional to the number of viable Mycobacteria in the medium. Different growth indicator have been used for the assay such as tetrazolium salts: XTT [2,3-bis- (2-methoxy-4-nitro-5-sulfophenyl)-2H-tetrazolium5-carboxanilide] and MTT [3(4,5-dimethylthiazol-2-yl)-2,5-diphenyltetrazoliumbromide] and the redox indicators Alamar blue and resazurin [19].

Colorimetric methods of drug susceptibility testing produce results more quickly than standard culture methods and are less costly than molecular methods. The average time to have first results was between 7 and 14 days compared with the reference standard method which takes 3 - 6 weeks. An example being the resazurin microtiter assay (REMA), which is based on a redox reaction which induces a blue to pink colour change in the presence of live bacteria [20].

Colorimetric nitrate reductase-based antibiotic susceptibility or CONRAS is a nitrate reductase-based test (NRA) for M. tuberculosis in Middlebrook 7H9 broth cultures. It is an indirect assay carried out on a solid media, with the media being supplemented by potassium and sodium nitrate at $1000 \mathrm{mg} / \mathrm{l}$ concentration to function as a growth indicator. This method employs the reduction of nitrate to nitrite by $M$. tuberculosis using the nitrate reductase enzyme which is detected by a reagent (Griess reagent) which turns a pink-purple colour [21].

These tests, however, have limitations such as Mycobacteria other than M. tuberculosis can produce cord factor, in MTT assay isoniazid can interfere with formazan production giving rise to false-resistant results. In these tests the use of liquid medium in a micro-titre plate format could also prove to be disadvantageous due to possible contamination between wells as well as for being a biohazard [22].

\section{Microscopic observation drug susceptibility}

Developed by a research team in Lima, Peru microscopic observation drug susceptibility (MODS) is a highly sensitive/specific assay for rapid and economic detection of Mycobacterium tubercu- 
losis and DST directly from sputum. This is a test reserved to two drugs isoniazid and rifampicin in regards to drug susceptibility testing. The workings of this test as explained by Linwei Wang., et al. is as follows, a 24-well plate with 4 wells allotted for each patient specimen is used, two of the wells contain RIF and INF and the other two are drug-free. The plates are sealed after inoculation and incubated M. tuberculosis is grown rapidly in liquid medium. The morphological characterization patterns specific to $M$. tuberculosis is employed for diagnosis following inoculation under an inverted microscope.

MODS has the advantage of having the ability to be used as an absolute concentration technique and it detects difficult rifampicin-borderline strains better than commercial liquid culture systems.

Despite being quite affordable and an effective alternative for testing sputum samples of TB-suspected individuals to existing gold standard liquid mycobacterial culture methods this method has its own drawbacks. This method has its shortcomings in resource-limited settings due to its concern regarding biosafety and efficiency in handling a large number of samples. Concerns in association with biosafety are due to usage of liquid wells in MODs assay, there is a risk of aerosolization, spillage, cross-contamination, and occupational infection as there is manipulation carried out of live liquid cultures, though sealed plates which do not require reopening might reduce this risk. This method also requires individual wells to be read manually posing a requirement of both time and human resources. There might also be difficulty about MOD's ability to discern $M$. tuberculosis and non-tuberculosis mycobacterium $[23,24]$.

\section{Molecular assays for drug resistance detection}

Line probe assay

The emergence of multidrug-resistant tuberculosis has expressed a formidable challenge forcing researchers to come up with swifter methods of detection due to the complex diagnostic and treatment obstacles. LPA is a rapid drug susceptibility detection test approved by WHO for first and second-line agents which can be used for testing of culture isolates (indirect testing) and also for smear microscopy positive specimens of acid-fast bacilli (AFB) as well as including both smear-positive and negative sputum specimens.
The primary principle of LPA is based on the reverse hybridization of the DNA on a strip. The detection is based on the binding of amplicons (DNA amplification products) to probes targeting the first and second-line agents affiliated to most common resistance mutation and to probes targeting the complementary wild-type DNA sequence. The detection of mutation is carried out by observing the pattern of binding of amplicon and the lack of hybridization of the amplicons to the corresponding wild type probes.

In case of the drug rifampicin, the mode of action of rifampicin is by binding to the beta-subunit of the RNA polymerase (coded for by rpoB gene) which in turn inhibits the protein transcription. Despite there being more than 50 mutations characterised by automated DNA sequencing codons 516,526 , or 531 involve major mutations. RIF-resistant TB can also be considered as a good surrogate marker for MDR-TB as more than $90 \%$ of RIF resistant TB is also resistant to INH. These advancements have therefore helped make further development of various methods for rapid detection of RIF-resistance conferring mutations, one of them being line probe assay.

The LPA kit consists of 10 oligonucleotide probes i.e. 5 of $\mathrm{S}$ probes (overlapping wild-type) and $4 \mathrm{R}$ probes (resistant genotype) for detection of specific mutations and a specific probe for M. tuberculosis complex immobilised on nitrocellulose paper strips. Direct clinical samples or extracted DNA samples are used to perform LPA by PCR amplification of the RIF- resistance-determining region of the rpoB gene. The immobilised probes are then hybridised with biotinylated PCR products and the results determined by colorimetric method. If a positive signal is retrieved from the wild-type $\mathrm{S}$ probe and negative from $\mathrm{R}$ probe the $M$. tuberculosis isolate is considered RIF susceptible. RIF resistance can be deliberated by the absence of one or more wild-type $S$ probe or a positive reaction obtained from one of the four R probes (Morgan, Kalantri, Flores, and Pai, 2005).

The first line-line probe assay GenoType MTBDRplus (referred to as GenoType MTBDRplus V1) was endorsed by the World Health Organisation in the year 2008 for the rapid detection of multidrugresistant tuberculosis. Newer versions have been subsequently developed since 2011 of the LPA technology, including) the GenoType MTBDRplus version 2 (referred to as GenoType MTBDRplus V2), and (ii) the Nipro NTM+MDRTB detection kit 2 (referred to as "Nipro", Tokyo, Japan). 
Regardless of being approved by WHO, line probe assay has its own limitations. Resistance cannot be ruled out entirely even in the existence of all WT probes as mutations which are responsible for conferring resistance are outside the region covered by the test thus necessitating auxiliary phenotypic DST to provide a full assessment. Identification of mutations can be inferred by the specific MUT probes or by the absence of amplicons binding to wild type probes. However, the presence of synonymous and nonsynonymous mutations (e.g. phylogenetic mutations) could cause systematic errors. Another one of the shortcomings is the inability of the test to detect resistant bacteria if the resistant population is less than $95 \%$ of the total bacterial population. The cost of the LPA kit also renders the test impractical for widespread use in those regions of the world most affected by MDR-TB and most in need of a method for its rapid diagnosis [25].

Real time PCR assays

Xpert MTB/RIF

Another DST approved by WHO in 2011 for the initial diagnosis of MDR-TB suspected individuals is the Xpert MTB/RIF [26]. The Xpert MTB/RIF is a fully automated, cartridge based, heminested real-time polymerase chain reaction (PCR) analysis. The cartridge is manufactured from plastic, is multichambered and contains all the reagents for sample processing and the subsequent real-time PCR run $[27,28]$. The analysis is carried out on the GeneXpert, Cepheid platform which is a software-driven cartridge processor and integrated fluorescence-based quantitative thermal cycler [28,29].

In this assay, amplification of aforementioned rpoB gene is carried out to analyze the region for mutations in the rifampin resistance-determining region (RRDR). The cartridge contains five molecular beacons for detecting these mutations. If at least two of the five probes are positive, then the samples are positive for the presence of $M$. tuberculosis and failure of one or more beacons to hybridize with the rpoB amplicon can be inferred as rifampicin resistance [27].

While there are several advantages of the Xpert MTB/RIF test such as short time required for the results and minimal technical training required for handling the system [30], there are few drawbacks too. One of the drawbacks is that the assay could overestimate MDR-TB in regions of RIF mono-resistance since it only detects RIF resistance [31].

\section{LATE-PCR with lights-on/lights-off probes}

Though the above mentioned diagnostic techniques are well established and approved by WHO, newer technologies are always being invented and tested to overcome existing drawbacks and to increase efficiency. One such new technique is LATE-PCR with Lights-On/Lights-Off Probes. This PCR technique has been described as an enhanced non-symmetric PCR technique (uses limiting and excess primers to generate larger amount of singlestranded amplicons). The technique uses two pairs of Lights-On/ Lights-Off probes of the same fluorescent colour to detect mutations in specific regions of the generated single-stranded amplicons. The Lights-On probe is labelled with a fluorophore and quencher while the Lights-Off probe is labelled only with a quencher. The LightsOff probe binds to a sequence adjacent to the Lights-On probe and absorbs the energy from the fluorophore. Since the probes have a short length, variance of even one base pair results in different fluorescent signatures. This is useful to assess any mutations in the DNA. As the signal from each Lights-On probe is extinguished, multiple probe pairs of the same colour or different colours can be utilized to analyse sequences several hundred nucleotides in length [32].

This new technique has been used to develop a new diagnostic tool called FluoroType MTBDR VER1.0 by Hain lifesciences. This technique can detect mutations in $r p o B$, katG, and inhA regions in a single tube. It has the advantage of hands-on time, faster results (within 3h), no DNA contamination, and automatic result interpretation when compared to GenoType MTBDRplus and has comparable sensitivity and specificity of RIF resistance detection to Genotype MTBDRplus and Xpert MTB/RIF. However, sensitivity and specificity of INH resistance detection is lower than GenoType MTBDRplus [33]. The tool has been further developed to produce a newer model called FluoroType MTBDR VER2.0 [34].

\section{Sequencing}

Susceptibility testing has undergone a prodigious innovation with its leap to molecular methods of resistance detection especially in relation to the development of various molecular tests endorsed by WHO such as Xpert MTB/RIF and the MTBDRplus, MTBDRsl, and Nipro line probe assays [35]. One of the best technologies for the rapid analysis of the genotype of an organism is sequencing. Targeted whole genome sequencing or Xpert Ultra (Cepheid) and 
Next Generation Sequencing (NGS) has the ability to provide both diagnosis for an individual patient and dispense mutation-specific information and phylogenetic data hence becoming a major breakthrough in molecular biology [33,35].

This allows an opening of attractive options of monitoring the surveillance of drug resistance by examination of all loci, delivering information in regard of changes both small or large in the genome, predict evolution of organism, detect epidemics thus helping in the management of patients with MDR-TB and provides guidance for suitable drug regimen selection $[35,36]$.

Despite the availability of several nucleic acid based assays each have their own sets of drawbacks such as the Genotype MTBDR plus method has the ability to only detect MDR strains via characterising mutations in katG gene (S315T), rpoB gene (D516V, H526Y/D, and S531L) and inhA promoters failing to detect XDR $M$. tuberculosis strains. The LPA method despite being both specific and sensitive can never reach $100 \%$ sensitivity as many mutations associated with resistance are yet to be discovered [37]. LPA also fails to detect some drug-resistant isolates mutations present in the genome of a minor population in case of co-infection [38]. A study conducted has also found that WGS has better performance than LPA in prediction of phenotypic DST in terms of sensitivity (94.2\% vs. 84.0\%) [39]. Therefore, various NGS-based kits are now available in the market one of them being the Ion Torrent Personal Genome Machine (PGM) a rapid (2 days) full length Mycobacterium tuberculosis gene analysis equipment following a novel protocol developed by Life technology [37].

In middle- and low- income countries there are various challenges of large-scale sequencing. Sequencing as in such requires robust software and database tools for the complete exploitation of this technology, the experiments conducted, data acquiesced and analysed needs specialised personnel and bioinformatics facilities for proper functioning, adding to this is the high GC content and repetitive nature of the genome of M. tuberculosis making sequencing quite challenging. This technology requires high amounts and high-quality DNA and determination of whether new mutations can confer anti-TB drug resistance. The major drawback for NGS in middle- and low- income countries is the heavy expenses presented by NGS platforms $[35,36,40]$.
Phage amplified biologically assay (PhaB assay)

A range of rapid molecular assays is available commercially for the detection of mutation associated with multi-drug resistance M. tuberculosis. However, these assays are impractically expensive, complex and have 90 - 95\% predictive. Such assays also harbour the drawback of being less predictive of resistance for drugs other than rifampicin and isoniazid. In the study conducted by IJ Eltringham., et al. PhaB assay was extended to the drugs ethambutol, pyrazinamide, streptomycin, and ciprofloxacin for 157 isolates in comparison to resistance ratio method retrieving significantly better correlations for ciprofloxacin, ethambutol, and pyrazinamide. The turn-around time for this assay is 2 - 3 days in comparison to the 10 days required for resistance ratio method [41].

This assay is based on the principle of the ability of $M$. tuberculosis to prevent the inactivation of mycobacteriophage from phagicidal chemicals thus protecting it. Therefore, when incubated with drugs the mycobacteriophage can only be protected by viable $M$. $t u$ berculosis that is, resistant bacilli. The viable bacilli protecting the phage within are then lysed after the rapid cycle of infection and replication of the phage. Lysis is observed as clear areas or plaques on a rapidly growing lawn of $M$. smegmatis [41,42].

The phage amplified biologically (PhaB) assay was developed by Wilson., et al. using D29 to detect viable M. tuberculosis, demonstrating the blocking ability of rifampicin to halt productive infection in sensitive strain but not in resistant [43].

In comparison to the BACTEC system, the PhaB assay has about one-sixth of the reagent cost. It also cuts down the requirement of purchasing and maintenance of high-cost monitoring equipment. Further no hazard or costs are incurred in regard to handling of radioactive material [44-47].

\section{Conclusion}

Drug-susceptibility testing is a widely practiced ritual followed to successfully treat tuberculosis patients and for the progression of developing new and effective strategies to overcome the hurdles faced due to the emergence of drug-resistant tuberculosis. Most people who develop TB can be cured with a timely diagnosis and treatment with antibiotics curtailing the onward transmission. As a result, various methods have been developed over the years for 
better and rapid results starting with the very first solid media method developing to the contemporary molecular methods. The current methods have been incapable of retrenching or meeting the dire needs of the populace thus further advancements in the future could aid to erode the statistical millions to a few hundred deaths per year. This demands the instigation of newer methods with advantages allowing accurate, easy, swift and an economic upper-hand to the global population.

\section{Bibliography}

1. Chakravorty S., et al. "Genotypic susceptibility testing of Mycobacterium tuberculosis isolates for amikacin and kanamycin resistance by use of a rapid sloppy molecular beacon-based assay identifies more cases of low-level drug resistance than phenotypic Lowenstein-Jensen testin". Journal of Clinical Microbiology 53.1 (2015): 43-51.

2. World health organization (WHO). "Global tuberculosis report 2019" (2019).

3. Kim K., et al. "A low cost/low power open source sensor system for automated tuberculosis drug susceptibility testing". Sensors (Switzerland) 16.6 (2016).

4. Campbell PJ., et al. "Molecular Detection of Mutations Associated with First-and Second-Line Drug Resistance Compared with Conventional Drug Susceptibility Testing of Mycobacterium tuberculosis † $\S$ ". Antimicrobial Agents and Chemotherapy 55.5 (2011): 2032-2041.

5. Kim SJ. "Controversial Issues in Tuberculosis” (2004).

6. Diriba G., et al. "Performance of Mycobacterium Growth Indicator Tube BACTEC 960 with Lowenstein-Jensen method for diagnosis of Mycobacterium tuberculosis at Ethiopian National Tuberculosis Reference Laboratory, Addis Ababa, Ethiopia”. BMC Research Notes 10.1 (2017).

7. Martin A., et al. "Multicentre laboratory validation of the colorimetric redox indicator (CRI) assay for the rapid detection of extensively drug-resistant (XDR) Mycobacterium tuberculosis". Journal of Antimicrobial Chemotherapy 66.4 (2011): 827833.
8. Riska PF., et al. "Rapid film-based determination of antibiotic susceptibilities of Mycobacterium tuberculosis strains by using a luciferase reporter phage and the Bronx Box". Journal of Clinical Microbiology 37.4 (1999): 1144-1149.

9. Martin A., et al. "Colorimetric redox-indicator methods for the rapid detection of multidrug resistance in Mycobacterium tuberculosis: A systematic review and meta-analysis". Journal of Antimicrobial Chemotherapy 59.2 (2007): 175-183.

10. Canetti G., et al. "Mycobacteria: Laboratory Methods for Testing Drug Sensitivity and Resistance" (1963).

11. van Klingeren, B., et al. "Drug Susceptibility Testing of Mycobacterium tuberculosis Complex by Use of a High-Throughput, Reproducible, Absolute Concentration Method". Journal of Clinical Microbiology 45.8 (2007): 2662-2668.

12. World health organization (WHO). Guidelines for surveillance of drug resistance in tuberculosis $5^{\text {th }}$ Edition (2015).

13. Acharya S., et al. "Comparison of Proportion and Resistance Ratio Methods for Drug Susceptibility Testing of Mycobacterium tuberculosis Isolated from Patients Attending National Tuberculosis Centre, Nepal”. SAARC Journal of Tuberculosis, Lung Diseases and HIV/AIDS 5.1 (2010): 13-20.

14. Jhamb SS., et al. "Determination of the activity of standard anti-tuberculosis drugs against intramacrophage Mycobacterium tuberculosis, in vitro: MGIT 960 as a viable alternative for BACTEC 460". Brazilian Journal of Infectious Diseases 18.3 (2014): 336-340.

15. Huang TS., et al. "Antimicrobial susceptibility testing of Mycobacterium tuberculosis to first-line drugs: comparisons of the MGIT 960 and BACTEC 460 systems". Annals of Clinical and Laboratory Science 32.2 (2002): 142-147.

16. Bemer P., et al. "Multicenter evaluation of fully automated BACTEC mycobacteria growth indicator tube 960 system for susceptibility testing of Mycobacterium tuberculosis". Journal of Clinical Microbiology 40.1 (2002): 150-154. 
17. Yajko DM., et al. "Colorimetric Method for Determining MICs of Antimicrobial Agents for Mycobacterium tuberculosis". Journal of Clinical Microbiology (1995).

18. Martin A., et al. "Colorimetric redox-indicator methods for the rapid detection of multidrug resistance in Mycobacterium tuberculosis: A systematic review and meta-analysis". Journal of Antimicrobial Chemotherapy 59.2 (2007): 175-183.

19. Katawera V., et al. "Evaluation of the modified colorimetric resazurin microtiter plate-based antibacterial assay for rapid and reliable tuberculosis drug susceptibility testing". BMC Microbiology 14.1 (2014): 259.

20. Bwanga F., et al. "Direct susceptibility testing for multi drug resistant tuberculosis: A meta-analysis". BMC Infectious Diseases 9.1 (2009): 67.

21. Syre H., et al. "Rapid Colorimetric Method for Testing Susceptibility of Mycobacterium tuberculosis to Isoniazid and Rifampin in Liquid Cultures". Journal of Clinical Microbiology 41.11 (2003): 5173-5177.

22. Kam KM. "Microscopic observation drug susceptibility (mods): Where are we going?" International Journal of Tuberculosis and Lung Disease 18.2 (2014): 127.

23. Wang L., et al. "Evaluating the Auto-MODS Assay, a Novel Tool for Tuberculosis Diagnosis for Use in Resource-Limited Settings" (2015).

24. Line probe assays for drug-resistant tuberculosis detection Interpretation and reporting guide for laboratory staff and clinicians. (n.d.).

25. WHO. Automated Real-Time Nucleic Acid Amplification Technology for Rapid and Simultaneous Detection of Tuberculosis and Rifampicin Resistance: Xpert MTB/RIF Assay for the Diagnosis of Pulmonary and Extrapulmonary TB in Adults and Children: Policy update. World Health Organisation (2013): 1-79.

26. Helb D., et al. "Rapid detection of Mycobacterium tuberculosis and rifampin resistance by use of on-demand, near-patient technology". Journal of Clinical Microbiology 48.1 (2010): 229237.
27. Raja S., et al. "Technology for Automated, Rapid, and Quantitative PCR or Reverse Transcription-PCR Clinical Testing". Clinical Chemistry 51.5 (2005): 882-890.

28. Boehme CC., et al. "Rapid molecular detection of tuberculosis and rifampin resistance". The New England Journal of Medicine 363.11 (2010): 1005-1015.

29. National Center for HIV., et al. "A New Tool to Diagnose Tuberculosis: The Xpert MTB/RIF Assay What is the Xpert MTB/RIF Assay? How Does the Xpert MTB/RIF Assay Work?".

30. Gu Y., et al. "Xpert MTB/RIF and GenoType MTBDRplus assays for the rapid diagnosis of bone and joint tuberculosis". International Journal of Infectious Diseases 36 (2015): 27-30.

31. Rice JE., et al. "Fluorescent signatures for variable DNA sequences". Nucleic Acids Research 40.21 (2012): e164-e164.

32. Nguyen TNA., et al. "Molecular Diagnosis of Drug-Resistant Tuberculosis; A Literature Review." Frontiers in Microbiology 10 (2019): 794.

33. FluoroType MTBDR | Detection of M. tuberculosis and its resistances. (n.d.).

34. Dheda K., et al. "The epidemiology, pathogenesis, transmission, diagnosis, and management of multidrug-resistant, extensively drug-resistant, and incurable tuberculosis". The Lancet Respiratory Medicine 5.4 (2017): 291-360.

35. Phelan J., et al. "The variability and reproducibility of whole genome sequencing technology for detecting resistance to anti-tuberculous drugs". Genome Medicine 8.1 (2016): 132.

36. Daum LT., et al. "Next-generation ion torrent sequencing of drug resistance mutations in Mycobacterium tuberculosis strains". Journal of Clinical Microbiology 50.12 (2012): 38313837.

37. Genestet C., et al. "Whole-genome sequencing in drug susceptibility testing of Mycobacterium tuberculosis in routine practice in Lyon, France". International Journal of Antimicrobial Agents (2020): 105912. 
38. Quan TP., et al. "Evaluation of Whole-Genome Sequencing for Mycobacterial Species Identification and Drug Susceptibility Testing in a Clinical Setting: a Large-Scale Prospective Assessment of Performance against Line Probe Assays and Phenotyping". Journal of Clinical Microbiology 56.2 (2018).

39. Zignol M., et al. "Genetic sequencing for surveillance of drug resistance in tuberculosis in highly endemic countries: a multi-country population-based surveillance study". The Lancet Infectious Diseases 18.6 (2018): 675-683.

40. Galí N., et al. "Use of a mycobacteriophage-based assay for rapid assessment of susceptibilities of Mycobacterium tuberculosis isolates to isoniazid and influence of resistance level on assay performance". Journal of Clinical Microbiology 44.1 (2006): 201-205.

41. DeRisi Joseph., et al. " $\beta$-sheet breaker peptides inhibit fibrillogenesis in a rat brain model of amyloidosis: Implications for Alzheimer's therapy". Nature 4 (1996): 822-826.

42. Eltringham IJ., et al. "Evaluation of a bacteriophage-based assay (phage amplified biologically assay) as a rapid screen for resistance to isoniazid, ethambutol, streptomycin, pyrazinamide, and ciprofloxacin among clinical isolates of Mycobacterium tuberculosis". Journal of Clinical Microbiology 37.11 (1999): 3528-3532.

43. Khan ZA., et al. "Current and emerging methods of antibiotic susceptibility testing". Diagnostics 9 (2019).

44. Kim SJ. "Drug-susceptibility testing in tuberculosis: Methods and reliability of results". European Respiratory Journal 25.3 (2005): 564-569.

45. Molecular line probe assays for rapid screening of patients at risk of multidrug-resistant tuberculosis (mdr-tb) policy statement (2008).

46. Siddiqi SH. Becton Dickinson Diagnostic Instruments System. Maryland, USA. BACTEC TB system. Product and procedure manual (1996).

\section{Assets from publication with us}

- Prompt Acknowledgement after receiving the article

- Thorough Double blinded peer review

- Rapid Publication

- Issue of Publication Certificate

- High visibility of your Published work

Website: www.actascientific.com/

Submit Article: www.actascientific.com/submission.php

Email us: editor@actascientific.com

Contact us: +919182824667 